\title{
In Vivo Imaging of Cerebrospinal Fluid Transport through the Intact Mouse Skull using Fluorescence Macroscopy
}

Sweeney, Amanda M.; Pla, Virginia; Du, Ting; Liu, Guojun; Sun, Qian; Peng, Sisi; Plog,

Benjamin A.; Kress, Benjamin T.; Wang, Xiaowei; Mestre, Humberto; Nedergaard, Maiken

Published in:

Journal of Visualized Experiments

DOI:

$10.3791 / 59774$

Publication date:

2019

Document version

Publisher's PDF, also known as Version of record

Document license:

Other

Citation for published version (APA):

Sweeney, A. M., Pla, V., Du, T., Liu, G., Sun, Q., Peng, S., Plog, B. A., Kress, B. T., Wang, X., Mestre, H., \& Nedergaard, M. (2019). In Vivo Imaging of Cerebrospinal Fluid Transport through the Intact Mouse Skull using Fluorescence Macroscopy. Journal of Visualized Experiments, (149), [e59774]. https://doi.org/10.3791/59774 


\title{
Video Article \\ In Vivo Imaging of Cerebrospinal Fluid Transport through the Intact Mouse Skull using Fluorescence Macroscopy
}

\author{
Amanda M Sweeney ${ }^{* 1}$, Virginia Plá ${ }^{1}$, Ting Du ${ }^{1}$, Guojun Liu ${ }^{1}$, Qian Sun ${ }^{1}$, Sisi Peng ${ }^{1}$, Benjamin A. Plog ${ }^{1}$, Benjamin T. Kress ${ }^{1}$, Xiaowei Wang ${ }^{1}$, \\ Humberto Mestre ${ }^{1}$, Maiken Nedergaard ${ }^{1,2}$ \\ ${ }^{1}$ Center for Translational Neuromedicine, University of Rochester Medical Center \\ ${ }^{2}$ Center for Translational Neuromedicine, University of Copenhagen \\ * These authors contributed equally
}

Correspondence to: Humberto Mestre at Humberto_Mestre@urmc.rochester.edu, Maiken Nedergaard at maiken_nedergaard@urmc.rochester.edu

URL: https://www.jove.com/video/59774

DOI: doi:10.3791/59774

Keywords: Neuroscience, Issue 149, transcranial, macroscopic, intact skull, in vivo imaging, glymphatic system, cerebrospinal fluid

Date Published: 7/29/2019

Citation: Sweeney, A.M., Plá, V., Du, T., Liu, G., Sun, Q., Peng, S., Plog, B.A., Kress, B.T., Wang, X., Mestre, H., Nedergaard, M. In Vivo Imaging of Cerebrospinal Fluid Transport through the Intact Mouse Skull using Fluorescence Macroscopy. J. Vis. Exp. (149), e59774, doi:10.3791/59774 (2019).

\section{Abstract}

Cerebrospinal fluid (CSF) flow in rodents has largely been studied using ex vivo quantification of tracers. Techniques such as two-photon microscopy and magnetic resonance imaging (MRI) have enabled in vivo quantification of CSF flow but they are limited by reduced imaging volumes and low spatial resolution, respectively. Recent work has found that CSF enters the brain parenchyma through a network of perivascular spaces surrounding the pial and penetrating arteries of the rodent cortex. This perivascular entry of CSF is a primary driver of the glymphatic system, a pathway implicated in the clearance of toxic metabolic solutes (e.g., amyloid- $\beta$ ). Here, we illustrate a new macroscopic imaging technique that allows real-time, mesoscopic imaging of fluorescent CSF tracers through the intact skull of live mice. This minimally-invasive method facilitates a multitude of experimental designs and enables single or repeated testing of CSF dynamics. Macroscopes have high spatial and temporal resolution and their large gantry and working distance allow for imaging while performing tasks on behavioral devices. This imaging approach has been validated using two-photon imaging and fluorescence measurements obtained from this technique strongly correlate with ex vivo fluorescence and quantification of radio-labeled tracers. In this protocol, we describe how transcranial macroscopic imaging can be used to evaluate glymphatic transport in live mice, offering an accessible alternative to more costly imaging modalities.

\section{Video Link}

The video component of this article can be found at https://www.jove.com/video/59774/

\section{Introduction}

Cerebrospinal fluid (CSF) bathes the brain and spinal cord and is involved in maintaining homeostasis, supplying nutrients, and regulating intracranial pressure ${ }^{1}$. CSF in the subarachnoid space enters the brain through a network of perivascular spaces (PVS) surrounding cortical pial arteries and then flows down along penetrating arterioles ${ }^{2}$. Once in the parenchyma, CSF exchanges with interstitial fluid (ISF), carrying harmful metabolites such as amyloid- $\beta(A \beta)$ and tau protein aggregates out of the brain through low resistance white matter tracts and perivenous spaces $^{2,3}$. This pathway is dependent on astroglial aquaporin-4 (AQP4) channels and has therefore been termed the glial-lymphatic (glymphatic) system ${ }^{4}$. Waste products of the neuropil are ultimately cleared from the CSF-ISF through lymphatic vessels near cranial nerves and in the meninges out towards the cervical lymph nodes ${ }^{5}$. The failure of this system has been implicated in several neurologic diseases such as Alzheimer's disease $^{6,7}$, traumatic brain injury ${ }^{3}$, and ischemic and hemorrhagic stroke ${ }^{8}$.

CSF transport can be visualized by infusing tracers into the cisterna magna $(\mathrm{CM})^{9,10}$ and glymphatic studies in the past have mainly utilized two-photon microscopy ${ }^{4,11,12,13}$, magnetic resonance imaging (MRI) 14,15,16,17, and ex vivo imaging ${ }^{3,6,11,18}$ to evaluate tracer kinetics. Twophoton microscopy is a suitable method for detailed imaging of CSF tracers in PVSs and the parenchyma due to its high spatial resolution, however, it has a narrow field of view and requires an invasive cranial window or thinning of the skull. Ex vivo imaging, in combination with immunohistochemistry, enables multilevel analyses ranging from single cells up to the whole brain ${ }^{19}$. However, the process of perfusion-fixation that is required to observe the post-mortem tissue produces profound changes in CSF flow direction and collapses the PVS, significantly altering the distribution and the location of the tracers ${ }^{12}$. Finally, while MRI can track CSF flow throughout the entire murine and human brain, it lacks spatial and temporal resolution of perivascular flow.

A new technique, transcranial macroscopic imaging, solves some of these limitations by enabling wide-field imaging of perivascular CSF transport in the entire dorsal cortex of living mice. This type of imaging is done with an epifluorescent macroscope using a multiband filter cube, tunable LED light source, and high-efficiency CMOS camera ${ }^{10}$. These set-ups are able to resolve PVSs up to 1-2 mm below the skull surface and can detect fluorophores up to 5-6 mm below the cortical surface while leaving the skull entirely intact ${ }^{10}$. Multiband filters and LEDs that can 
quickly tune the excitation wavelength enable the use of multiple fluorophores allowing CSF to be labeled with tracers of different molecular weights and chemical properties in the same experiment.

This procedure requires a simple, minimally invasive surgery to expose the skull and place a light-weight head plate to stabilize the head during the imaging session. Tracers can be delivered into the CM without drilling into the skull or penetrating the cortical tissue with pipettes or cannulas ${ }^{9,20}$. Both $\mathrm{CM}$ cannulas and head plates remain stable for several days to weeks and facilitate more complex experimental designs compared to the classical end-point visualization. This protocol describes how transcranial macroscopic imaging is used to study glymphatic system function following acute or chronic injection of fluorescent CSF tracer into the CM of anesthetized/sleeping or awake mice.

Protocol

All experiments were approved by the University Committee on Animal Resources (UCAR, Protocol No. 2011-023) at the University of Rochester and performed according to the NIH Guide for the Care and Use of Laboratory Animals.

\section{Preparing the cisterna magna cannula, head plate, and head holder}

1. Sterilize all surgical instruments and head plates before surgery. NOTE: Fluorescent tracers are delivered directly into CSF via a cisterna magna cannulation. For detailed instructions on this procedure, please refer to Xavier et al ${ }^{9}$.

2. Briefly, using a needle driver, break the tip of a $30 \mathrm{G} \times 12.7 \mathrm{~mm} \mathrm{(1/2} \mathrm{inch})$ needle, $3 / 4$ of the way down, and place the blunt end of the needle into one end of polyethylene 10 (PE10) tubing (about $45 \mathrm{~cm}$ long). Make sure that only the bevel is protruding out of the border of the PE10 tubing.

3. Break off $1 / 4$ of the beveled end of another $30 \mathrm{G}$ needle and place the blunt end of the remaining needle into the other end of the PE10 tubing, with the plastic Luer-lock still attached.

4. Fill a $100 \mu \mathrm{L}$ glass syringe with sterile, artificial CSF (aCSF: $126 \mathrm{mM} \mathrm{NaCl}, 2.5 \mathrm{mM} \mathrm{KCl}, 1.25 \mathrm{mM} \mathrm{NaH}_{2} \mathrm{PO}_{4}, 2 \mathrm{mM} \mathrm{MgSO}_{4}, 2 \mathrm{mM} \mathrm{CaCl}_{2}, 10$ $\mathrm{mM}$ glucose, and $26 \mathrm{mM} \mathrm{NaHCO}_{3}$ ).

5. Attach the syringe to the end of the line and fill it with aCSF until it reaches the tip of the beveled needle. It is important that the syringe is backfilled with aCSF and not air, as an air column is more prone to variable infusion volumes.

6. For chronic experiments, leave the line filled with aCSF and skip Step 1.7.

7. For acute experiments, place the syringe on an infusion pump and withdraw approximately $5 \mathrm{~mm}$ of air, which will prevent mixing. Afterwards, withdraw the total volume of tracer(s) that is desired for the experiment (20\% extra is recommended due to dead space losses).

NOTE: The PE10 tubing should be long enough so that the air bubble does not enter the plastic cuff of the glass syringe when loading the tracer. For a typical experiment, the protocol uses $10 \mu \mathrm{L}$ of bovine serum albumin conjugated to Alexa Fluor 647 (BSA-647) diluted in aCSF at $0.5 \%$.

8. Confirm that the head plate fits into the head holder and that it is the right size for the mouse being used. Anatomical markers to ensure this are: the top border of the window aligns with the interocular line and the posterior border falls rostral to the occipital crest. NOTE: Stainless-steel head plates can be sterilized and reused. Most cyanoacrylate mixtures can be removed with an acetone solution.

\section{Surgical procedure}

1. Weigh and anesthetize the mouse (e.g. ketamine/xylazine; $100 \mathrm{mg} / \mathrm{kg}$ ketamine, $10 \mathrm{mg} / \mathrm{kg}$ xylazine; i.p.).

2. Once the mouse no longer responds to a toe pinch, moisten the neck and head with sterile water and shave using clippers. Once the area is shaved, wipe the area with an alcohol swab again to remove any residual hair.

NOTE: Moistening the fur before clipping dramatically reduces the amount of hair in the imaging window after the incision.

3. Place the mouse in a stereotactic frame on top of a temperature-controlled pad and apply petroleum ophthalmic ointment to the mouse's eyes to ensure they do not dry out.

4. Clean the exposed skin with a chlorhexidine swab. After $2 \mathrm{~min}$, remove the chlorhexidine with an alcohol wipe. Finally, apply an iodine solution which can be left to dry.

5. Inject subcutaneous analgesia $(0.25 \%$ Bupivacaine $\mathrm{HCl})$ to the top of the skull and the neck.

6. Starting on the part of the neck that covers the occipital crest, make a midline cut in the overlying skin and continue rostrally towards the interorbital line. Incise laterally to the border where the temporal muscle inserts into the skull. Remove all of the skin of the fusiform incision to expose both the frontal and parietal bones.

CAUTION: The retroorbital sinus lies caudal to the eyes and large branches of the posterior facial vein lie rostral to the pinna. Be careful when making the incision to spare these structures. If this occurs, stop bleeding by maintaining hemostasis with a sterile cotton swab for several minutes and continue.

7. Irrigate the skull with sterile saline and clean the surface using cotton swabs so that it is free of debris and hair since these will interfere with the image quality. Skull transparency is best preserved by leaving the periosteum and overlying fascia intact.

NOTE: If these structures are accidentally removed, the skull can become dry and opaque over time. Re-moisten with aCSF or use a mixture of paraffin oil and glycerol to reduce skull reflectance in acute experiments ${ }^{21}$.

8. Proceed to inserting the cisterna magna cannula - for surgical details of this procedure, refer to Xavier et al ${ }^{9}$.

9. After inserting the CM cannula, apply a mixture of dental cement with cyanoacrylate glue to the ventral side of the head plate around the border and place it on the skull so that the anterior border of the head plate aligns with the posterior tip of the nasal bone and the posterior border aligns with the anterior aspect of the interparietal bone, making sure that the sagittal suture (midline) is centered and straight relative to the window (Figure 1B).

10. Fix the position of the head plate using a couple drops of glue accelerator. Fill any remaining gaps with the cement mixture and cure it with accelerator.

CAUTION: Make sure the cyanoacrylate does not come in contact with the mouse's eyes or block the imaging window.

11. Glue the CM cannula to the head plate to ensure that these do not become detached during transport or when the mouse wakes up. 
12. For acute experiments, skip to Step 2.16.

13. For chronic experiments, apply a thin layer of clear-drying cyanoacrylate glue to the exposed skull, being careful not to create bubbles as these will interfere with the imaging. The glue provides protection for the skull and will not interfere with the imaging. Make sure that the glue covers the exposed skull all the way to the skin at the incision border.

14. Use a hemostat clamp to hold the aCSF-filled PE10 tubing 2-3 cm from the $\mathrm{CM}$ and cut the line with a high-temperature cautery tip. Once it is separated, flatten the melted PE10 tubing to seal the cannula.

CAUTION: Make sure not to release the clamp until after the cannula has been sealed and confirm there are no leaks in the tubing to prevent a CSF fistula.

15. Administer carprofen ( $5 \mathrm{mg} / \mathrm{kg}$ every $24 \mathrm{~h}$ for 3 days; i.p. or s.c.) and return the mouse to a temperature-controlled, single-housed cage and allow it to recover for at least $24 \mathrm{~h}$ prior to imaging. Do not leave the mouse unattended until it regains sufficient consciousness to maintain sternal recumbency. Intact cranial windows remain stable for several weeks. NOTE: The chronic experiment can be paused here.

CAUTION: Some postoperative complications are: cephalohematoma/subgaleal hematomas, CSF fistula, and infection.

16. For acute experiments, place the mouse into the head holder using the head plate so the skull is in a fixed position. Make sure to check anesthesia level and place a heating pad under the mouse. The mouse is now ready to be taken to the macroscope.

CAUTION: Carefully transport the mouse together with the infusion pump on a cart. If the CM cannulation becomes dislodged, it will cause a drop in intracranial pressure and any CSF leaks will alter the experimental results.

\section{Preparing the mouse for imaging}

NOTE: The protocol varies depending on whether the imaging experiment will be performed on an anesthetized (start at Step 3.1) or awake (start at Step 3.2) mouse.

1. Anesthetized Mice

1. Place the head holder on the stage of the macroscope, making sure there are no kinks in the line from the syringe pump to the CM cannula and that it is not taut since this could affect the tracer infusion.

2. Observe respiratory rate and pink coloration of the mucous membranes, indicating good oxygenation. Inject saline subcutaneously to secure hydration level if necessary. Check to make sure the animal is sufficiently anesthetized and re-dose if needed.

3. Turn on the macroscope camera and LED and start LIVE mode.

4. Confirm the magnification of the imaging field so that the nasofrontal suture at the top of the field and the lambdoid suture at the bottom can clearly be visualized, with the sagittal suture parallel and centered to the middle of the image (Figure 1C). Once in place, secure the head holder to the macroscope stage with tape.

5. Focus the macroscope on the exposed skull. Despite macroscopes having a relatively large depth of field, the curvature of the mouse's skull only allows for a specific area to be in focus. Best results are obtained when the focal plane is located on the lateral sides of the parietal bones posterior to the coronal sutures.

NOTE: This is the location of the middle cerebral arteries (MCA), where most CSF inflow occurs (Figure 1D,E) ${ }^{10}$

2. Awake Mice

1. Prior to the imaging session, let the animals recover for at least $24 \mathrm{~h}$ from the head plate surgery. Longer recovery periods (5-7 days) are also recommended. During this time, train the mouse to be head fixed on the stage in the restraint tube for $0.5-1 \mathrm{~h} \mathrm{per} \mathrm{day} \mathrm{for} \mathrm{the}$ duration of the recovery period.

NOTE: Habituation allows the mouse to be attached to the head holder without anesthesia and reduces stress and anxiety during the actual experiment. If this is not feasible and anesthesia does not interfere with the experiment, an induction dose of an inhaled anesthetic (e.g., isoflurane $2 \%$ at $1-2 \mathrm{~L} / \mathrm{min}_{2}$ flow rate) can be used to quickly attach the mouse to the head stage.

2. Once the mouse is fixed to the head holder and in the restraint tube, follow Steps 3.1.1-3.1.5.

\section{Infusion of fluorescent CSF tracers}

\section{Acute CM Cannulation}

1. Since the tracer was already loaded into the cannula before being placed in the cisterna magna in Step 1.6, set the infusion pump to the desired rate and volume. Infusion paradigms routinely used are $5-10 \mu \mathrm{L}$ at $1-2 \mu \mathrm{L} / \mathrm{min}$, but these parameters can be adjusted depending on the particular experiment, or the size and age of the animal.

\section{Chronic CM Cannulation}

1. Prior to beginning the imaging session, follow Steps 1.2-1.7 for acute experiments to prepare the infusion line to deliver the tracers.

2. Once the line is prepared, using a hemostat clamp cut the sealed end of the chronic CM cannula. Take the needle from the line prepared in Step 4.2.1 and gently insert it into the cannula. Release the clamp and using the syringe pump, advance the CSF tracer at the desired infusion rate (e.g., $2 \mu \mathrm{L} / \mathrm{min}$ ) for the experiment until the tracer reaches the implanted needle in the CM.

CAUTION: If the needle pierces through the tubing when connecting the line, remove the needle, cut the pierced segment and repeat this step. Any tear in the PE10 tubing will cause a leak and affect the results of the experiment.

\section{Setting up the Imaging Session}

1. Based on the fluorescent tracer being infused, determine the excitation wavelength and the exposure time for each channel. Choose the shortest exposure time necessary to visualize the tracer in order to maximize the temporal resolution of the time-lapse imaging. This exposure time will be used for all subsequent experiments aiming to compare CSF transport between different animals. 
NOTE: One tip is to use the tracer in the CM line to adjust the exposure time. Although this is a useful first approach, this should be optimized over time.

2. Choose the duration of the experiment and the intervals at which the images will be acquired. Experiments normally last between 30-60 min depending on what phase of glymphatic transport is of interest. Frame rates of 1 frame/min and faster are sufficient for most experiments.

3. Set the file name and the saving directory.

4. If the imaging will be collected in addition to simultaneous acquisition of other variables (e.g., electrocardiogram, arterial blood pressure, electrophysiology), the macroscope and pump can be programmed to be triggered with the data acquisition software. Check that the triggering function on the macroscope is correct before moving to the next step.

\section{Transcranial optical imaging experiment}

1. Start the tracer infusion and the imaging at the same time.

2. Routinely check the CM cannula and the PE10 tubing for any signs of a leak. If there is any tracer leaking at the CM, the results from this experiment must be excluded.

NOTE: If tracer starts to accumulate in the cerebellum and does not travel the glymphatic pathway of the MCA, it is likely that the CM cannula was injected into the cerebellum. This data should not be included.

3. For acute experiments, after completion of the experiment, remove the mouse from the microscope and check that it is still adequately anesthetized. Quickly decapitate the mouse and harvest the brain tissue. Immersion-fix the brain in 4\% paraformaldehyde (PFA) overnight at $4{ }^{\circ} \mathrm{C}$.

1. For chronic experiments, once the imaging is completed, remove the CM line from the cannula, flush with sterile aCSF and reseal the $\mathrm{CM}$ cannula with a high-temperature cautery tip. Remove the mouse from the head stand and return it to its cage. Repeat this process until further imaging is not required and then follow Step 6.3.

\section{Data analysis}

NOTE: Matlab-based analyses, such as CSF front-tracking can extract large amounts of quantitative data from the tracer fronts in these imaging datasets ${ }^{10,22}$. However, these file types can also be easily imported and analyzed in open-source image analysis software like Fiji ${ }^{23}$.

1. Import the image stacks into Fiji using the Bio-Formats import tool. This function will preserve the file metadata which includes the time and pixel resolution. Save the image stack as a .tiff file.

2. Manually draw a region of interest (ROI) around the skull or the area of interest using the polygon selection tool. For example, in Figure 1G an ROI was drawn separately for the ipsilateral and for the contralateral hemisphere after a traumatic brain injury. Make sure to add the ROI into the ROI Manager (Analyze>Tools>ROI Manager) and save it. NOTE: CSF transport can be quantified in two main ways: 1) mean fluorescence intensity over time or 2) influx area expressed as a percentage of the ROI or total area $\left(\mathrm{mm}^{2}\right)$ after having applied a threshold. The latter method (Figure 1H) will be described in the next steps.

3. Set the threshold on the frame with maximal fluorescence (Select Image>Adjust $>$ Threshold...). Automated threshold methods such as Otsu are normally good at detecting CSF tracer. Apply the threshold.

4. Before going forward, make sure that in Analyze>Set Measurements, the options Area and Area Fraction are selected. Then in the RO Manager, select More>Multi Measure.

5. Convert the \%Area value into $\mathrm{mm}^{2}$ using the Area value of the ROI from the output. The \%Area values reflect the percent of the dorsal cortical surface with CSF tracer.

6. Plot CSF tracer influx in $\mathrm{mm}^{2}$ as a function of time.

\section{Representative Results}

CSF influx is imaged on an epifluorescent macroscope (Figure 1A), which allows for mesoscopic imaging of CSF tracer transport in the murine cortex. The whole-skull head plate permits the visualization of the rostral nasal bones, both frontal and parietal bones in the center, and the rostral portion of the interparietal bone caudally (Figure 1B). During imaging, the nasofrontal, sagittal, coronal, and lambdoid sutures can be readily identified (Figure 1C). Once the infusion of CSF tracer into the CM begins (Figure 1D), tracer fluorescence is first seen in large pools of subarachnoid CSF at the basal cistern, olfactofrontal cistern, and the quadrigeminal cistern near the pineal recess (Figure 1E, left). CSF tracers then enter the brain along perivascular spaces of the cortical pial branches of the middle cerebral artery (MCA) (Figure 1E, right).

Transcranial optical imaging can be used to study glymphatic function after traumatic brain injury (TBI) ${ }^{3}$. Mice received a moderate TBI and immediately afterwards a fluorescent CSF tracer (BSA-647) was injected into the $\mathrm{CM}^{24}$. CSF transport was imaged for 60 min after TBI (Figure 1F). Macroscopic imaging shows that tracer is first seen at the olfactofrontal cistern but glymphatic influx along cortical PVSs is completely abolished on the side of the TBI (Figure 1G, Supp. Movie 1). The inhibitory effect of TBI on glymphatic function has been shown using several other tracer quantification methods and could underlie the relationship between TBI and the accumulation of $A \beta$ and tau seen after injury ${ }^{3}$. Quantitative analysis of in vivo images show that ipsilateral influx area is decreased nearly a third compared to the contralateral hemisphere (Figure 1H). 
А. смов

Camera

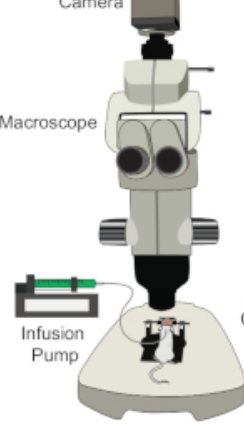

F.

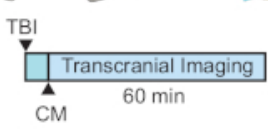

G.

Injection

B.

D.

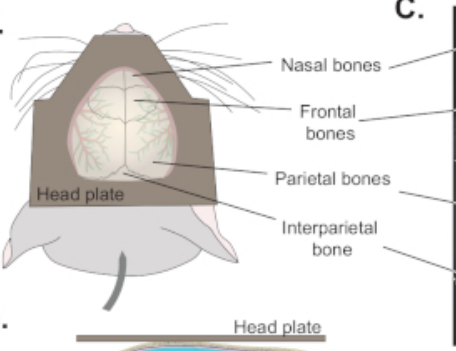

C.
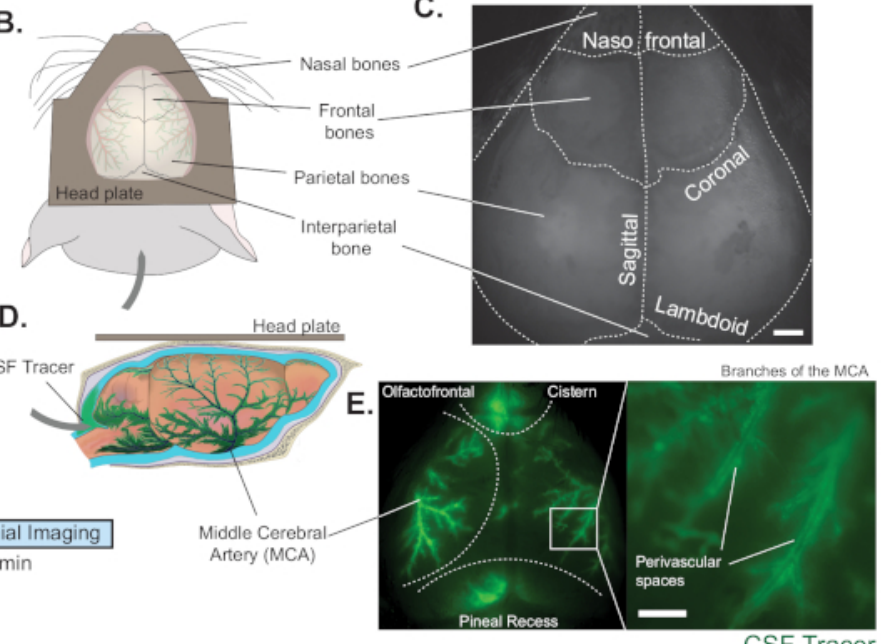

CSF Tracer

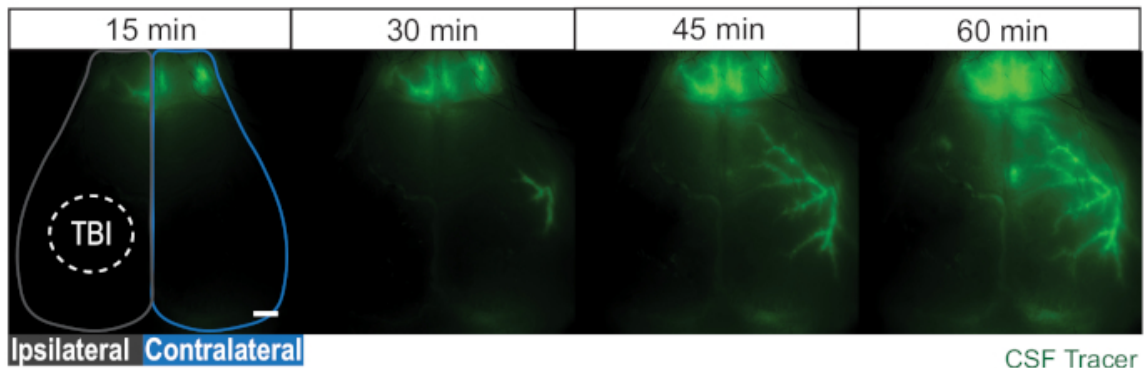

H.

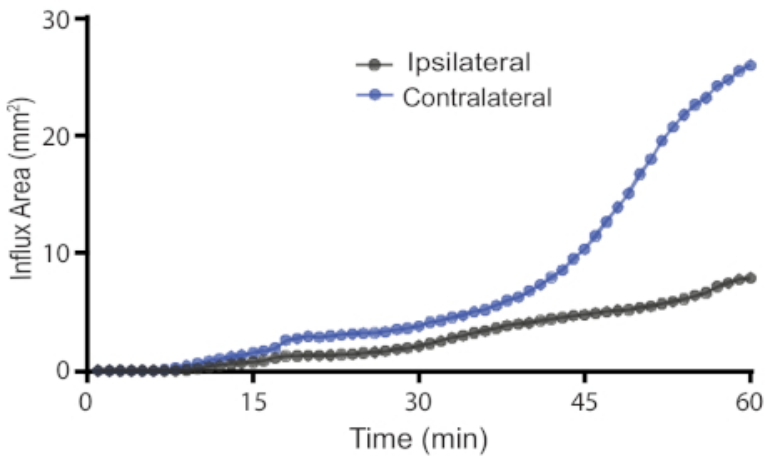

Figure 1. Transcranial macroscopic imaging. (A) Schematic of the macroscopic imaging set up. (B) Dorsal view of the position of the head plate on the skull. The interparietal bone and the nasal, frontal, and parietal bones are all visible. (C) An exposed mouse skull during imaging before CSF tracer has appeared, clearly showing all cranial sutures of the intact skull. Scale bar: $1 \mathrm{~mm}$ (D) Schematic of a lateral view of CSF tracer entering the cisterna magna (CM) and traveling from the basal cistern along the glymphatic pathway. (E, left panel) Macroscopic imaging of glymphatic influx in a ketamine-xylazine anesthetized wildtype mouse at 20 minutes post CM injection (BSA-647; $10 \mu \mathrm{L}$ at $2 \mu \mathrm{L} /$ $\mathrm{min}$ ). CSF tracer is seen in the olfactofrontal cistern around the rostral rhinal vein below the nasofrontal suture, along some parts of the superior sagittal sinus below the sagittal suture, and in the pineal recess surrounding the transverse sinus below the lambdoid suture. (E, right panel) Digital magnification of the image on the left shows the high spatial resolution obtained with these microscopes. CSF tracer travels within the perivascular spaces of the middle cerebral artery $(\mathrm{MCA})^{10}$. Scale bar: $0.5 \mathrm{~mm}(\mathbf{F})$ Experimental timeline. An anesthetized wildtype mouse received a moderate traumatic brain injury (TBI) ${ }^{24}$ and immediately after a CM injection (BSA-647; $10 \mu \mathrm{L}$ at $2 \mu \mathrm{L} / \mathrm{min}$ ), followed by 60 min of macroscopic imaging. (G) Time-lapse images of CSF tracer transport after TBI (dashed line). Scale bar: $1 \mathrm{~mm}(\mathbf{H})$ Influx area ( $\mathrm{mm}^{2}$ ) over each hemisphere during the 60-min experiment, quantified from the ROIs in $(G)$, the hemisphere that received TBI (ipsilateral) and the hemisphere that did not (contralateral). Please click here to view a larger version of this figure.

Supplemental Figure 1. Blueprint of custom head plate. (A, B) Exact measurements (in $\mathrm{mm}$ ) of the custom head plate used in the transcranial macroscopic imaging protocol. 3D schematics of a hollowed out head plate (C) and a whole head plate (D). Please click here to download this file.

Supplemental Movie 1. Time-lapse imaging of CSF transport after moderate traumatic brain injury to the left parietal bone. Duration: 60 min. Scale bar: $1 \mathrm{~mm}$ Please click here to download this file. 


\section{Discussion}

We have described a detailed protocol for performing transcranial CSF imaging in live mice using commercially-available fluorescent macroscopes and tracers. This technique is simple and minimally-invasive, yet quantitative. In vivo imaging correlates well with sensitive methods such as liquid scintillation counting of radio-labeled tracers including ${ }^{3} \mathrm{H}$-dextran and ${ }^{14} \mathrm{C}$-inulin after $\mathrm{CM}$ delivery, and with ex vivo coronal section quantification ${ }^{10,18}$. Validation with two-photon microscopy demonstrates that CSF tracers seen along cortical blood vessels under the macroscope are primarily located within perivascular spaces of the MCA and its branches ${ }^{10}$. These CSF inflow pathways are a critical component of the glymphatic system ${ }^{19}$. Although not covered in this protocol, these set-ups can also be used to image CSF clearance pathways such as the meningeal and cervical lymphatics ${ }^{25,26}$.

Improvements in head plate design enables chronic, stable, wide-field imaging of the same mouse over time. Head plate shape, size, and weight can be tailored for each specific application. With advances in laser cutting and 3D printing, there are few limitations as to the specifications. Head plating also allows for longitudinal imaging of either anesthetized or awake animals and the ability to image through an intact skull avoids neuroinflammation and edema associated with the placement of cranial or thin skull windows ${ }^{27,28,29}$. This is an important advantage since stereotaxic delivery of fluorescent tracers through the cortex into the striatum or lateral ventricles using a cranial burr hole greatly reduce glymphatic function ${ }^{20,26}$. The large gantry and working distances of most commercial macroscopes allow for mice to be fixed to the stage in various configurations, including running wheels or floating mazes. Mice can be trained and habituated to being head fixed to the microscope stage during the 5-7-day recovery period to facilitate awake imaging ${ }^{30}$.

One of the principal limitations of this method is the low penetration depth of the macroscope compared to two-photon microscopy ${ }^{10}$. This device is capable of resolving only a few millimeters of the cortical surface below the intact skull. However, while this limits the imaging of processes occurring deeper down in the tissue, it is generally not a problem for glymphatic analyses since the major routes of entry are primarily located around the main arteries of the dorsal cortical surface. Despite being unable to resolve deeper structures, macroscopes with high efficiency scientific CMOS cameras have great fluorescence detection; despite tracer not being located at the brain surface, total fluorescence intensity correlates with the amount of tracer found in the brain at every time point after the start of the CM injection ${ }^{10}$. These parameters are improved by the use of far-red, near-infrared, or infrared tracers since imaging at these longer wavelengths reduces tissue auto-fluorescence and the scattering of light, and have a superior signal-to-noise ratio than lower emission wavelength fluorophores. The standard macroscope enables imaging of more than one tracer in the same experiment. Depending of the configuration of the macroscope, the filter turret has to rotate between acquisitions, drastically reducing the temporal resolution that can be obtained. This can be improved by the use of tunable LEDs and a multiband filter cube. Despite this improvement, multichannel imaging is not truly simultaneous, since there is a delay in the acquisition while the LED switches between excitation wavelengths. It is possible to achieve simultaneous dual channel imaging using image splitting optics that are compatible with most macroscopic set-ups; however, these sacrifice spatial resolution by dividing the full resolution of the CMOS camera (2048 $\times 2048$ pixel) into two fields of view with half the resolution (1024 x 1024 pixel). While CMOS cameras are quite adequate for this utilization, the use of a CCD camera can be applied to this application as well. An additional factor that reduces temporal resolution is the exposure time required for adequate excitation of the chosen fluorophore, which normally ranges between $50-1000 \mathrm{~ms}$. This can be further optimized by using either $2 \times 2$ or $4 \times 4$ pixel binning, which reduces the exposure time and increases the frame rate, at the expense of spatial resolution. Despite these limitations, transcranial macroscopic multichannel imaging is capable of achieving frame rates between $10-20 \mathrm{~Hz}$ with high spatial resolution.

Recent examples in transcranial macroscopic imaging have used aquaporin-4 (AQP4) knock out mice ${ }^{10,20}$ and platelet-derived growth factor $\mathrm{B}$ (PDGF-B) retention motif knockout mice ${ }^{22}$ to demonstrate the importance of AQP4 and PDGF-B in the glymphatic system. The studies used C57BI6 wildtype mice to compare to the knockout mouse lines. They transcranially imaged the mice for a duration of 30 min using fluorescent tracers and the results concluded that the knockout lines had dramatically reduced CSF influx compared to wildtypes.

These properties make transcranial optical imaging an ideal technique to study CSF transport, especially between 2 or more cohorts of animals. It permits measurements of intracisternal tracer kinetics in live mice at a micron scale resolution, at a fraction of the cost of other in vivo imaging modalities. This technique allows the study of the glymphatic system in a physiological, non-invasive way, and has been useful in helping elucidate some of the factors that regulate its function in health and disease ${ }^{10,20,25}$. However, its most exciting attribute is its potential to answer future questions about CSF hydrodynamics.

\section{Disclosures}

The authors have nothing to disclose.

\section{Acknowledgments}

This work was funded by the National Institute of Neurological Disorders and Stroke and the National Institute on Aging (US National Institutes of Health; R01NS100366 and RF1AG057575 to MN), the Fondation Leducq Transatlantic Networks of Excellence Program, and the EU Horizon 2020 research and innovation programme (grant no. 666881; SVDs@target). We would also like to thank Dan Xue for expert assistance with graphic illustrations.

\section{References}

1. Tumani, H., Huss, A., \& Bachhuber, F. The cerebrospinal fluid and barriers - anatomic and physiologic considerations. Handbook of Clinical Neurology. 146 21-32, (2017). 
2. Jessen, N. A., Munk, A. S., Lundgaard, I., \& Nedergaard, M. The Glymphatic System: A Beginner's Guide. Neurochemical Research. 40 (12), 2583-2599, (2015).

3. Iliff, J. J. et al. Impairment of glymphatic pathway function promotes tau pathology after traumatic brain injury. The Journal of Neuroscience. 34 (49), 16180-16193, (2014).

4. Iliff, J. J. et al. A paravascular pathway facilitates CSF flow through the brain parenchyma and the clearance of interstitial solutes, including amyloid beta. Science Translational Medicine. 4 (147), 147ra111, (2012).

5. Louveau, A. et al. Structural and functional features of central nervous system lymphatic vessels. Nature. 523 (7560), 337-341, (2015)

6. Peng, W. et al. Suppression of glymphatic fluid transport in a mouse model of Alzheimer's disease. Neurobiology of Disease. 93 215-225, (2016).

7. Da Mesquita, S. et al. Functional aspects of meningeal lymphatics in ageing and Alzheimer's disease. Nature. 560 (7717), 185-191, (2018).

8. Gaberel, T. et al. Impaired glymphatic perfusion after strokes revealed by contrast-enhanced MRI: a new target for fibrinolysis? Stroke. 45 (10), 3092-3096, (2014).

9. Xavier, A. L. R. et al. Cannula Implantation into the Cisterna Magna of Rodents. Journal of Visualized Experiments. 10.3791/57378 (135), (2018).

10. Plog, B. A. et al. Transcranial optical imaging reveals a pathway for optimizing the delivery of immunotherapeutics to the brain. JCI Insight. 3 (23), (2018).

11. Kress, B. T. et al. Impairment of paravascular clearance pathways in the aging brain. Annals of Neurology. 76 (6), 845-861, (2014).

12. Mestre, H. et al. Flow of cerebrospinal fluid is driven by arterial pulsations and is reduced in hypertension. Nature Communications. 9 (1), 4878, (2018).

13. Xie, L. et al. Sleep drives metabolite clearance from the adult brain. Science. 342 (6156), 373-377, (2013).

14. Plog, B. A., \& Nedergaard, M. The Glymphatic System in Central Nervous System Health and Disease: Past, Present, and Future. Annual Review of Pathology. 13 379-394, (2018).

15. Iliff, J. J. et al. Brain-wide pathway for waste clearance captured by contrast-enhanced MRI. Journal of Clinical Investigation. 123 (3), 1299-1309, (2013).

16. Davoodi-Bojd, E. et al. Modeling glymphatic system of the brain using MRI. Neuroimage. 188 616-627, (2019).

17. Lee, H. et al. The Effect of Body Posture on Brain Glymphatic Transport. The Journal of Neuroscience. 35 (31), 11034-11044, (2015).

18. Hablitz, L. M. et al. Increased glymphatic influx is correlated with high EEG delta power and low heart rate in mice under anesthesia. Science Advances. 5 (2), eaav5447, (2019).

19. Rasmussen, M. K., Mestre, H., \& Nedergaard, M. The glymphatic pathway in neurological disorders. The Lancet Neurology. 17 (11), 1016-1024, (2018).

20. Mestre, H. et al. Aquaporin-4-dependent glymphatic solute transport in the rodent brain. Elife. 7, (2018).

21. Monai, H. et al. Calcium imaging reveals glial involvement in transcranial direct current stimulation-induced plasticity in mouse brain. Nature Communications. 7 11100, (2016).

22. Munk, A. S. et al. PDGF-B Is Required for Development of the Glymphatic System. Cell Reports. 26 (11), 2955-2969 e2953, (2019).

23. Schindelin, J. et al. Fiji: an open-source platform for biological-image analysis. Nature Methods. 9 (7), 676-682, (2012).

24. Ren, Z. et al. 'Hit \& Run' model of closed-skull traumatic brain injury (TBI) reveals complex patterns of post-traumatic AQP4 dysregulation. Journal of Cerebral Blood Flow \& Metabolism. 33 (6), 834-845, (2013).

25. Plog, B. A. et al. Biomarkers of traumatic injury are transported from brain to blood via the glymphatic system. The Journal of Neuroscience. 35 (2), 518-526, (2015).

26. Ma, Q., Ineichen, B. V., Detmar, M., \& Proulx, S. T. Outflow of cerebrospinal fluid is predominantly through lymphatic vessels and is reduced in aged mice. Nature Communications. 8 (1), 1434, (2017).

27. Roth, T. L. et al. Transcranial amelioration of inflammation and cell death after brain injury. Nature. 505 (7482), 223-228, (2014).

28. Xu, H. T., Pan, F., Yang, G., \& Gan, W. B. Choice of cranial window type for in vivo imaging affects dendritic spine turnover in the cortex. Nature Neuroscience. 10 (5), 549-551, (2007).

29. Ma, Q. et al. Rapid lymphatic efflux limits cerebrospinal fluid flow to the brain. Acta Neuropathologica. 137 (1), 151-165, (2019).

30. Silasi, G., Xiao, D., Vanni, M. P., Chen, A. C., \& Murphy, T. H. Intact skull chronic windows for mesoscopic wide-field imaging in awake mice. Journal of Neuroscience Methods. 267 141-149, (2016). 